\title{
Two Stage Sub-Wavelength Motion Correction in Human Microvasculature for CEUS Imaging
}

\author{
Sevan Harput ${ }^{1}$, Kirsten Christensen-Jeffries ${ }^{2}$, Yuanwei Li $^{1}$, Jemma Brown $^{2}$, \\ Robert J. Eckersley ${ }^{2 *}$, Chris Dunsby ${ }^{3 *}$, and Meng-Xing Tang ${ }^{1 *}$ \\ ${ }^{1}$ ULIS Group, Department of Bioengineering, Imperial College London, London, SW7 2BP, UK \\ ${ }^{2}$ Biomedical Engineering Department, Division of Imaging Sciences, King's College London, SE1 7EH, London, UK \\ ${ }^{3}$ Department of Physics and the Centre for Pathology, Imperial College London, London, SW7 2AZ, UK \\ ${ }^{*}$ These authors contributed equally to this work \\ E-mail: S.Harput@imperial.ac.uk, Mengxing.Tang@imperial.ac.uk
}

\begin{abstract}
The structure of microvasculature cannot be resolved using clinical B-mode or contrast-enhanced ultrasound (CEUS) imaging due to the fundamental diffraction limit at clinical ultrasound frequencies. It is possible to overcome this resolution limitation by localizing individual microbubbles through multiple frames and forming a super-resolved image. However, ultrasound super-resolution creates its unique problems since the structures to be imaged are on the order of $10 \mathrm{~s}$ of $\mu \mathrm{m}$. Tissue movement much larger than $10 \mu \mathrm{m}$ is common in clinical imaging, which can significantly reduce the accuracy of superresolution images created from microbubble locations gathered through hundreds of frames.

This study investigated an existing motion estimation algorithm from magnetic resonance imaging for ultrasound superresolution imaging. Its correction accuracy is evaluated using simulations with increasing complexity of motion. Feasibility of the method for ultrasound super-resolution in vivo is demonstrated on clinical ultrasound images. For a chosen microvessel, the super-resolution image without motion correction achieved a sub-wavelength resolution; however after the application of proposed two-stage motion correction method the size of the vessel was reduced to half.
\end{abstract}

\section{INTRODUCTION}

By localizing spatially isolated microbubbles with high precision through multiple ultrasound frames, an ultrasound super-resolution image is generated. Even at ultrasonic frequencies as low as $2-3 \mathrm{MHz}$, a localization precision of $2-4 \mu \mathrm{m}$ can be achieved with an unmodified clinical ultrasound system for stationary objects [1], [2]. This localization precision determines the maximum achievable resolution in super-resolution image that can be as high as of $10-20$ times the imaging wavelength.

Researchers demonstrated the use of ultrasound superresolution imaging in many different controlled experiments and pre-clinical studies [3]-[9]. For the applications that are closer to a clinical imaging setup, researchers reported the problems caused by motion artefacts. Ackermann and Schmitz tracked multiple microbubbles in a xenograft-bearing mouse tumour to measure the capillary blood flow. They have discarded 1151 frames out of 6000 acquired frames within four minutes due to the respiratory motion [7]. Lin et al. experienced a similar problem in tumour-bearing rats while performing 3D ultrasound localization microscopy. They have excluded $20-30 \%$ of the acquired frames in 11.5 minutes due to motion artefacts affecting the measured microbubble positions [8]. There are other methods based on localisation of spatially non-isolated microbubbles from a higher concentration. These methods can significantly reduce the acquisition duration; however they are still prone to motion artefacts at micrometre level [9], [10].

Ultrasound super-resolution imaging can easily achieve sub-wavelength resolution and localisation precision, but the problem of tissue motion must be addressed in order for it to be successful for clinical imaging. The motion should be compensated with an accuracy higher than the spatial resolution of the super-resolution image, which is usually around $10-20 \mu \mathrm{m}$. This study employed an existing motion estimation algorithm from magnetic resonance imaging to achieve the required motion correction accuracy and the results are demonstrated on clinical ultrasound images.

\section{Materials And Methods}

\section{A. Motion Correction}

Motion is an inherent part of diagnostic ultrasound imaging and it limits maximum achievable resolution in ultrasound super-resolution imaging. Therefore, motion correction is crucial for in vivo measurements. Rigid motion correction can be suitable for some modalities with a low spatial resolution. However, a super-resolved ultrasound frame is a combination of multiple acquisitions that usually take seconds to minutes to acquire. Over such a long duration, the motion between the initial and last frame will include local tissue deformations. Probe motion and respiratory motion is usually considered to be rigid, however human soft tissue is mostly anisotropic and tissue deformation is only in the linear elastic region for tissue strains up to $5 \%$ [11]. In addition to this, there are other motion sources that create local deformations such as pulsations of arterioles and venules, and twitching of muscles

In this study, an image registration model adopted from MRI is used for motion estimation. MATLAB functions are 


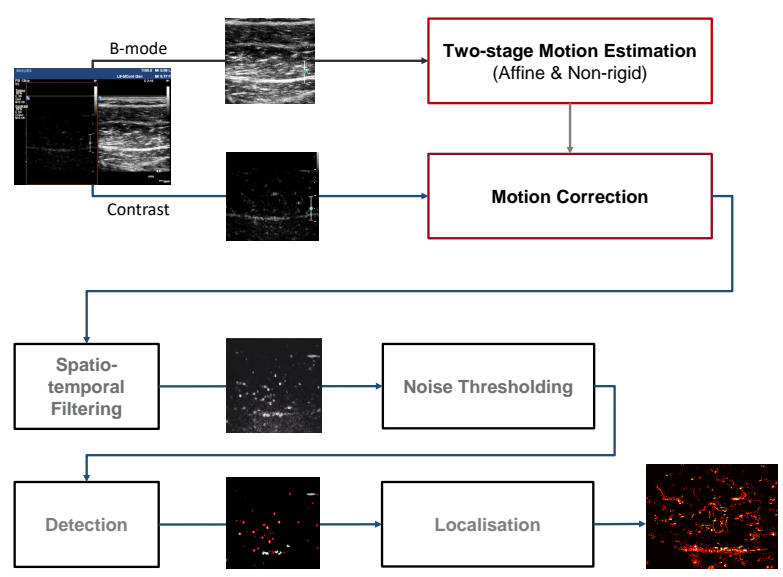

Fig. 1. Figure illustrates the processing chain of super-resolution imaging. Affine and non-rigid motion estimation are the first step.

available at [12]. The model is based on the work of Rueckert et al. and Lee et al. [13], [14] and it is capable of performing a rigid, affine, non-rigid, and a two stage motion estimation. This study uses the two stage motion estimation method, where the first stage is an affine estimation and the second stage is a non-rigid estimation. The affine registration estimates rigid motion such as translation and rotation and also shearing and resizing. The non-rigid registration is a B-spline based free-form deformation that can estimate the local compression and rarefaction of tissue [13]. Smoothness of the non-rigid estimation is crucial to capture the local deformation of the soft tissue. Therefore, a regularization parameter is used to achieve a smooth registration.

\section{B. Field II simulations}

To verify the MC accuracy, Field II simulations were performed with a homogeneous tissue phantom [15], [16]. Rigid tissue motion was generated by moving the location of scatterers together. Non-rigid motion was simulated by using a linear stress-strain relation and displacing the scatterers independently. In the axial direction, non-rigid motion was created by displacing the location of scatterers as a function of depth to mimic compression of tissue by the ultrasound probe. In the lateral direction, non-rigid motion was created by displacing the location of scatterers as a function of lateral distance to mimic muscle contraction in human lower limb. The maximum applied axial and lateral motion was $1 \mathrm{~mm}$. A new speckle pattern was created for each tested scenario. Simulation parameters were chosen specifically to match the parameters of the clinical study with a centre frequency $6 \mathrm{MHz}, 80 \%$ bandwidth, 160 elements and a pitch of $237.5 \mu \mathrm{m}$ and an attenuation coefficient of $0.5 \mathrm{~dB} / \mathrm{cm} / \mathrm{MHz}$. B-mode images were log-compressed and downsampled with a final pixel size of $60 \times 60 \mu \mathrm{m}^{2}$ and a dynamic range of $50 \mathrm{~dB}$.

\section{Clinical Data}

The tibialis muscles of healthy adult volunteers were scanned using a Philips iU22 ultrasound scanner (Philips Medical Systems, Bothell, WA) with a 3-9 MHz linear array
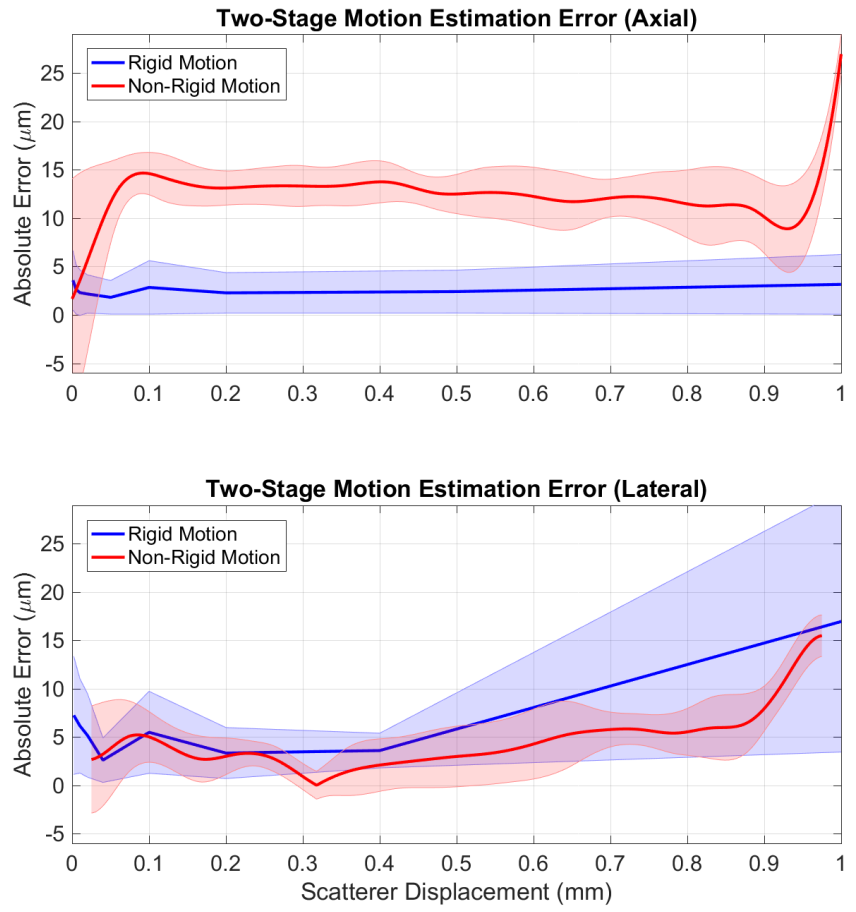

Fig. 2. Figure shows the motion estimation error for simulated rigid and nonrigid tissue motion in the axial and lateral directions. The absolute error values are plotted with a transparent shade that represents the standard deviation of 20 simulations with white Gaussian noise.

probe. B-mode and CEUS images were acquired during the injection of 4 times diluted Sonovue (Bracco S.p.A, Milan, Italy) microbubbles at an MI of 0.06. Data was recorded over a duration of 45 seconds with $585 \mathrm{~B}$-mode and CEUS images with a pixel size of $60 \times 60 \mu \mathrm{m}^{2}$ at a frame rate of $13 \mathrm{~Hz}$. As shown in Fig. 1 B-mode frames were used for motion estimation and the motion correction was performed on CEUS frames.

After motion correction, a singular value decomposition filter was used to remove the residual tissue echoes [17], [18] and a noise threshold was applied. In the detection stage multiple microbubble echoes were rejected and the last superlocalization stage was performed as explained in [19].

\section{RESUlts \& Discussion}

Results of the rigid and non-rigid motion simulations are shown in Fig. 2 for $20 \mathrm{~dB}$ SNR. The mean motion estimation error and standard deviation were better than $20 \mu \mathrm{m}$ for almost all of the simulated scenarios, which is far smaller than the $250 \mu \mathrm{m}$ wavelength and $60 \mu \mathrm{m}$ pixel size. The same set of simulations were also performed with $10 \mathrm{~dB}$ image SNR, where the error values increase above $50 \mu \mathrm{m}$. This value is not suitable for super-resolution imaging and thus not showed in this section.

After the feasibility study, the proposed two-stage motion estimation method was applied on the clinical B-mode images and the estimated motion was used to correct the CEUS images as explained in Fig. 1. 

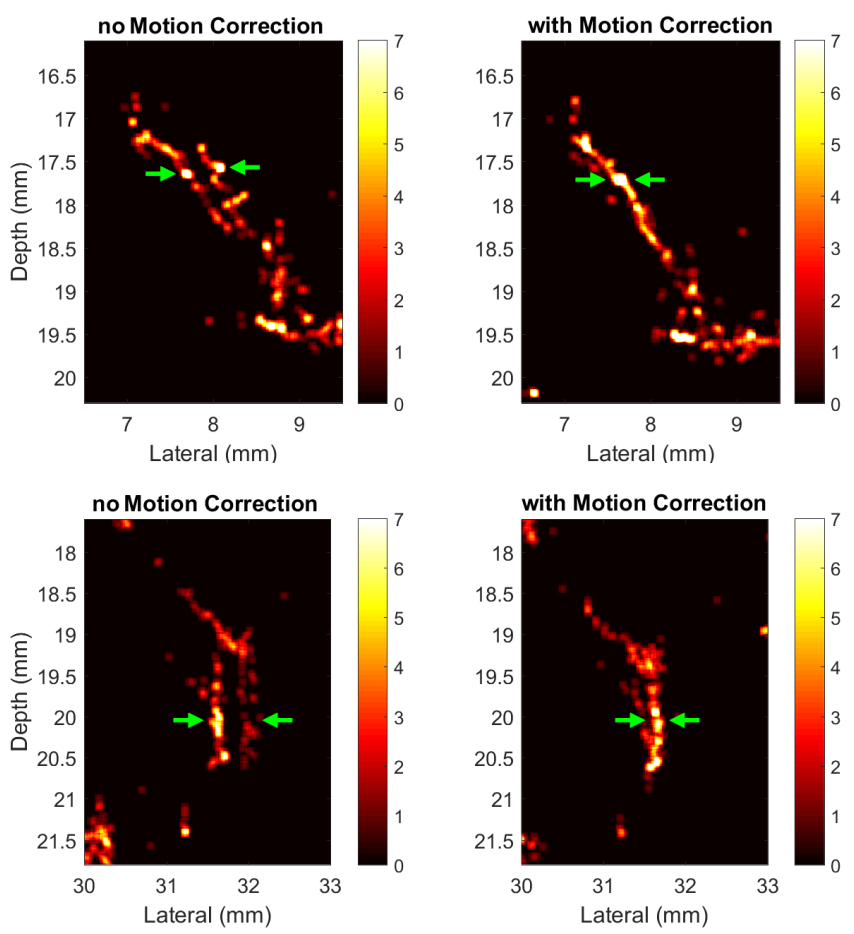

Fig. 3. Two examples of super-resolution images acquired in human lower limb are displayed with and without motion correction. Green arrows show the location of detected microvessels before and after motion correction.

It is hard to demonstrate the accuracy of the motion correction on a clinical dataset without the ground truth, but the simulation study shows that the proposed image registration method can achieve the required accuracy. The superresolution images of healthy volunteers after motion correction were evaluated qualitatively based on the quality improvement of the microvessels imaged. We evaluated vessel structures that were expected to be non-tortuous. The benefit of using two stage motion correction on super-resolution imaging in human microvasculature is illustrated in Fig. 3 with two examples. After motion correction the vessels with artificial shadows were visualised as single vessels.

Fig. 4 (top) demonstrates the effect of motion correction on a chosen vessel. The average thickness of the vessel inside the boxes are given in Fig. 4 (bottom). Sizes of the vessels from these images were measured by using the function described in [20]. The full-width at half maximum (FWHM) of the vessel was measured as $769 \mu \mathrm{m}$ from the maximum intensity projection of the CEUS image stack. The super-resolution image without motion correction achieved a sub-wavelength vessel FWHM of $227 \mu \mathrm{m}$; however after the application of proposed two-stage motion correction method the FWHM of the vessel was reduced to $115 \mu \mathrm{m}$.

\section{CONCLUSIONS}

Tissue motion correction is an ongoing challenge for all diagnostic ultrasound applications. This study employed an existing motion estimation algorithm from magnetic resonance imaging and demonstrated its feasibility for super-resolution
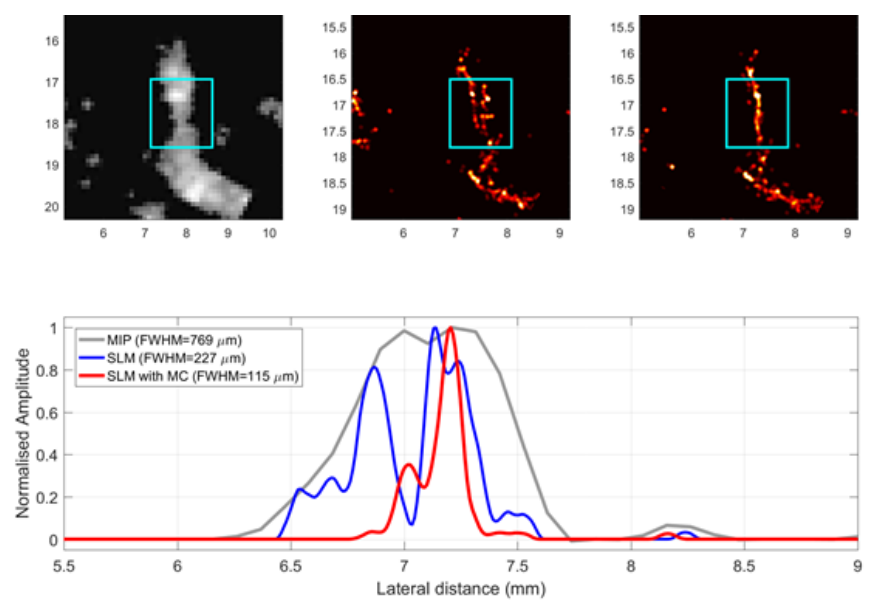

Fig. 4. (Top-left) Maximum intensity projection (MIP) of the CEUS image stack for the selected microvessel. (Top-middle) Super-resolution image without motion correction. (Top-right) Super-resolution image after motion correction. The blue rectangles show the area where the FWHM of the vessel was measured in the horizontal direction. (Bottom) Sizes of the vessels measured by averaging a cross-section of the images above. Grey colour represents the CEUS MIP, blue represents the super-resolution image without motion correction, and red represents the super-resolution image with motion correction.

ultrasound imaging. The proposed two-stage motion estimation method is a combination of affine image registration that can estimate the global motion, and non-rigid image registration that can estimate the local deformation of tissue. The first stage estimates for the large motion and provides a good initial condition for the second stage.

The motion estimation was performed on B-mode images, but the proposed motion correction method can be applied to RF data. The future goal of this study is to further improve the motion correction accuracy by exploiting the phase information in RF signal.

The proposed motion estimation method achieved an error smaller than $20 \mu \mathrm{m}$ for ultrasound images with $20 \mathrm{~dB}$ SNR, $60 \mu \mathrm{m}$ pixel size and wavelength of $250 \mu \mathrm{m}$. The subpixel and sub-wavelength motion correction made it possible to visualize microvascular structures by localizing spatially isolated microbubbles.

\section{ACKNOWLEDGMENT}

This work was supported by the Engineering and Physical Sciences Research Council (UK) via grant no. EP/N015487/1 and EP/N014855/1.

\section{REFERENCES}

[1] O. M. Viessmann, R. J. Eckersley, K. Christensen-Jeffries, M. X. Tang, and C. Dunsby, "Acoustic super-resolution with ultrasound and microbubbles," Phys. Med. Biol, vol. 58, pp. 6447-6458, 2013.

[2] Y. Desailly, J. Pierre, O. Couture, and M. Tanter, "Resolution limits of ultrafast ultrasound localization microscopy," Phys. Med. Biol, vol. 60, pp. 8723-8740, 2015.

[3] M. A. O. Reilly and K. Hynynen, "A super-resolution ultrasound method for brain vascular mapping," Medical Physics, vol. 40, no. 110701, 2013.

[4] Y. Desailly, O. Couture, M. Fink, and M. Tanter, "Sono-activated ultrasound localization microscopy," Applied Physics Letters, vol. 103, no. 174107,2013 
[5] K. Christensen-Jeffries, R. J. Browning, M.-X. Tang, C. Dunsby, and R. J. Eckersley, "In vivo acoustic super-resolution and super-resolved velocity mapping using microbubbles," IEEE Trans Med Imaging, vol. 34, no. 2, pp. 433-440, 2015.

[6] C. Errico, J. Pierre, S. Pezet, Y. Desailly, O. C. Zsolt Lenkei and, and M. Tanter, "Ultrafast ultrasound localization microscopy for deep superresolution vascular imaging," Nature, vol. 527, pp. 499-507, 2015.

[7] D. Ackermann and G. Schmitz, "Detection and tracking of multiple microbubbles in ultrasound b-mode images," IEEE Trans. Ultrason., Ferroelect., Freq. Control, vol. 63, no. 1, pp. 72-82, 2016.

[8] F. Lin, S. E. Shelton, D. Espindola, J. D. Rojas, G. Pinton, and P. A. Dayton, "3-d ultrasound localization microscopy for identifying microvascular morphology features of tumor angiogenesis at a resolution beyond the diffraction limit of conventional ultrasound," Theranostics, vol. 7, no. 1, pp. 196-204, 2017.

[9] A. Bar-Zion, C. Tremblay-Darveau, O. Solomon, D. Adam, and Y. C. Eldar, "Fast vascular ultrasound imaging with enhanced spatial resolution and background rejection," IEEE Trans Med Imaging, vol. 36, pp. 169-180, 2017.

[10] S. Harput, K. Christensen-Jeffries, J. Brown, R. J. Eckersley, C. Dunsby, and M.-X. Tang, "Localisation of multiple non-isolated microbubbles with frequency decomposition in super-resolution imaging," in IEEE International Ultrasonics Symposium (IUS), 2017, pp. 1-4.

[11] E. Chen, J. Novakofski, W. Jenkins, and W. O'Brien, "Young 's modulus measurements of soft tissues with application to elasticity imaging," Ultrasonics, Ferroelectrics and Frequency Control, IEEE Transactions on, vol. 43, no. 1, pp. 191-194, 1996.

[12] D.-J. Kroon. [Online]. Available: https://uk.mathworks.com/ matlabcentral/fileexchange/20057-b-spline-grid-image-and-point-basedregistration
[13] D. Rueckert, L. I. Sonoda, C. Hayes, D. L. G. Hill, M. O. Leach, and D. J. Hawkes, "Nonrigid registration using free-form deformations: Application to breast mr images," IEEE Transactions on Medical Imaging, vol. 18 , no. 8, pp. 712-721, 1999.

[14] S. Lee, G. Wolberg, and S. Y. Shin, "Scattered data interpolation with multilevel b-splines," IEEE Trans. Visualization Comput. Graph., vol. 3, pp. 228-244, 1997.

[15] J. Jensen and N. B. Svendsen, "Calculation of pressure fields from arbitrarily shaped, apodized, and excited ultrasound transducers," $U l$ trasonics, Ferroelectrics and Frequency Control, IEEE Transactions on, vol. 39, pp. 262-267, 1992.

[16] J. Jensen, "Field: A program for simulating ultrasound systems," in Medical \& Biological Engineering \& Computing, vol. 34, no. 1, 1996, pp. 351-353.

[17] C. Demene, T. Deffieux, M. Pernot, B.-F. Osmanski, V. Biran, J.-L. Gennisson, L.-A. Sieu, A. Bergel, S. Franqui, J.-M. Correas, I. Cohen, O. Baud, and M. Tanter, "Spatiotemporal clutter filtering of ultrafast ultrasound data highly increases doppler and fultrasound sensitivity," IEEE Trans Med Imaging, vol. 34, no. 11, pp. 2271-2285, 2015.

[18] J. Brown, K. Christensen-Jeffries, S. Harput, C. Dunsby, M.-X. Tang, and R. J. Eckersley, "Investigation of microbubble detection methods for super-resolution imaging of microvasculature," in IEEE International Ultrasonics Symposium (IUS), 2017, pp. 1-4.

[19] K. Christensen-Jeffries, S. Harput, J. Brown, P. N. T. Wells, P. Aljabar, C. Dunsby, M.-X. Tang, and R. J. Eckersley, "Microbubble axial localization errors in ultrasonic super-resolution imaging," IEEE Trans. Ultrason., Ferroelect., Freq. Control, p. in press, 2017.

[20] S. Harput, J. McLaughlan, D. M. Cowell, and S. Freear, "New performance metrics for ultrasound pulse compression systems," in Ultrasonics Symposium (IUS), 2014 IEEE International, 2014, pp. 440-443. 